Article

\title{
Analysis of Synchronization Stability for Multi VSCs Parallel-Connected to Weak Grids by Improved Net Damping Criterion
}

\author{
Dong Wang ${ }^{1}{ }^{\mathbb{D}}$, Xiaojie Zhang ${ }^{2}$, Lei Yang ${ }^{3}$, Yunhui Huang ${ }^{1, *}$, Wei Huang ${ }^{4}$, Chen $\mathrm{Wu}^{4}$ \\ and Shengnan $\mathrm{Li}^{3}$ \\ 1 Department of Electrical Engineering, School of Automation, Wuhan University of Technology, \\ Wuhan 430070, China; dongwang@whut.edu.cn \\ 2 State Key Laboratory of Advanced Electromagnetic Engineering and Technology, and School of \\ Electrical and Electronic Engineering, Huazhong University of Science and Technology, Wuhan 430074, \\ China; xjzhang16@hust.edu.cn \\ 3 Electric Power Research Institute of Yunan, Kunming 650217, China; 15911577929@139.com (L.Y.); \\ lsn788@sina.com (S.L.) \\ 4 Electric Power Dispatching and Control Center of Yunan, Kunming 650200, China; haxwell@163.com (W.H.); \\ wuchen@dltd.yn.csg.cn (C.W.) \\ * Correspondence: h.yunhui@whut.edu.cn; Tel.: +86-150-0274-9904
}

Received: 8 June 2020; Accepted: 25 June 2020; Published: 29 June 2020

\begin{abstract}
Recent studies show that the loss of stability for a voltage-source converter (VSC) in weak-grid connection is largely related to its synchronization unit, i.e., the phase-locked loop (PLL). This paper studies the synchronization stability of a system comprised by two VSCs in parallel connection to a weak grid. A reduced transfer function based small-signal model, which can allow for the interactions between PLL and converter outer power controls, is first proposed. Then, an improved net damping criterion is used to analyze the damping and stability characters of such system under various operating conditions and different controller configurations. Compared to the conventional net damping criterion, the used criterion has wider applicability in terms of stability judgment. Case studies show that the studied system tends to be unstable at weak-grid or heavy-loading conditions. The instability can be in the form of oscillations or monotonic divergence, in which, the latter is more likely to occur for the converters without grid voltage regulation capabilities. Besides, the net damping-based sensitivity studies can provide guidance on control tuning or design for stability enhancement. Detailed model-based time domain simulations are conducted to verify the analysis results.
\end{abstract}

Keywords: weak grid; VSC; PLL; net damping criterion; synchronization stability

\section{Introduction}

With the rapid development of renewable generation, large numbers of power electronics converters penetrate the power systems for energy conversion and transmission. These new devices also significantly change the dynamics and stability of power systems. The synchronization among the power electronics-based devices is one of the basic dynamics problems, drawing increased attention recently. Phased-locked loop (PLL) is the most widely used synchronization techniques for converters in single-phase as well as three phase systems applications [1,2]. Generally, PLL can well synchronize the converter with the power system under normal grid condition, but its performance is significantly affected by the grid voltage dynamics. In particular, weak-grid integration is one of the challenging 
operating conditions for PLL-synchronized converters, loss of stability has been reported in single voltage source converter (VSC) [3-7] under weak-grid conditions.

In the literature [3], a detailed single-input single output (SISO) model of a VSC considering the dynamics of inner current controls, outer power controls, and PLL is formulated. This model facilitates the control design of a VSC with adequate stability margins. In contrast, for the simplicity of analysis, reduced small-signal models covering the inner current control timescale and outer DC-link control timescale are also separately proposed in [4] and [5,6]. Literature [6,7] both find that the interactions between active power controls and PLL can lead to instability at weak-grid conditions in wind generation and high-voltage direct transmission (HVDC) systems. These results indicate that the dynamics of VSC outer power controls need to be modeled for synchronization stability studies.

Except for converter-grid interactions, synchronization stability involving the interactions with synchronous generator (SG) and other VSC-based devices has also caught increased attention recently. Literature [8] proposes a detailed impedance model of a VSC to study the subsynchronous interactions with a nearby SG; and reshaping techniques by modifying PLL dynamics or inserting active impedance are also put forward to improve the damping properties in the subsynchronous frequency range. Similar dq-frame impedance analysis was also carried out for a system comprising an inverter and a rectifier [9], which showed that higher bandwidths of PLL for the inverter can increase the frequency range of negative resistance. Besides, except for PLL, recent work [10] with impedance analysis identified that the effect of AC voltage control also strongly effects the stability of multi-STATCOM systems. The above works analyze the stability impact from the port characters of a device, some works also find that the control interactions between converters can be considerable for the overall system stability. As demonstrated in [11,12], serious control interactions among different terminals in VSC based multi-terminal direct current (MTDC) grids can lead to poorly damped oscillations. Literature [13] proposed a model for DC-link voltage stability analysis for a weak-grid-tied multi-VSC system, and eigenvalue analysis identified that two nearby VSCs can interact to cause instability when the grid strength is weak. A multi-input multi-output (MIMO) analysis based on the calculation of the norm of impedance was carried out to investigate the synchronization stability of a two converter system [14], and found that the bandwidths of PLL should be limited to avoid weak-grid instability. However, the model in [14] does not include the power controls. Besides, in literature [15], residue-based analysis is given to illustrate the synchronization instabilities due to the interaction of PLLs in two doubly-fed induction generator (DFIG) based wind generators.

Among the approaches for small-signal power systems stability studies, eigenvalue analysis is very popular for large-scale system analysis. This method can accurately determine system stability, but it formulates the studied system into a single closed-loop representation, which makes it hard to assess the interactions between subsystems, as well as evaluating the relative contribution of a subsystem to the overall stability [16]. In addition, the dq-frame impedance is also a preferred frequency-domain approach in power electronics studies, this method applies Nyquist stability criterion to analyze the converter-grid impedance ratio to check stability, and has been extensively used to study the current control stability of converters $[17,18]$. The Nyquist stability criterion essentially holds for single-input single-output (SISO) transfer function. However, when the PLL dynamics is included, the dq-impedance model of a VSC turns into MIMO form $[18,19]$, so the impedance criterion cannot be directly applied. The net damping criterion is another widely used frequency-domain tool. This method is initially proposed by Canay to study the torsional interactions for turbine-generator sets $[20,21]$, and it examines system stability in terms of the total net damping contribution from the electrical and mechanical systems at the resonance frequency. Compared to the MIMO analysis given in [14], this SISO-based approach has the advantage of providing an accurate evaluation of stability margin. Additionally, a system is divided into two parts in its analysis. Here come the primary merits of this method. It allows to extract a subsystem's contribution to the closed-loop stability based on the frequency-domain characters, as well as possibly locating the dominant stability factors [22]. However, as proven in [23], this classical criterion is not a sufficient and necessary criterion for stability 
examination under all the conditions, whether it can be directly applied for the synchronization stability analysis in multi-VSCs is not determined.

This paper will investigate the synchronization stability of two nearby VSCs in parallel connection to a weak grid by using a comprehensive net damping criterion. The objective of this study is to: (1) build a proper model for synchronization stability analysis of this system; (2) provide a full study on the impact of system parameters and subsystem interactions on stability in view of the net damping contribution. For interaction analysis purpose, the received power of a VSC are divided into two parts: one caused by the current perturbations of itself, and the other from the current perturbations of the other VSC. The damping contributions of the two parts will be comparably examined at various operating conditions. The main contributions of this work include:

(1) Building a reduced small-signal model suitable for synchronization stability analysis of multi-VSC systems. The used VSC module is much simpler than the detailed one in [3] for synchronization stability studies owing to the neglection of fast inner current controls, but more accurate compared to the models given in $[9,14]$ for counting the interactions between PLL and outer power controls.

(2) Comprehensively applying net damping criterion to investigate multi-VSC system's synchronization stability. The used criterion is a combination of the classical criterion propose by Canay [20] and an alternative one in our recent work [24], where the later can serve as a complement to enlarge the application feasibility of the classical criterion. The effectiveness of this method is confirmed by eigenvalue analysis. Additionally, with this method, the impact of various system parameters, including different grid strengths, power outputs and bandwidths of controllers, on closed-loop system stability are quantitatively examined.

The paper is organized as follows. A brief sketch of the classical net damping criterion is first given in Section 2, a discussion on its limitations is offered and a complemental criterion is proposed. Subsequently, a small-signal model for the studied system is built in Section 3. How to formulate the model to facilitate the use of net damping criterion will be illustrated. Then in Section 4, sensitivity studies at various operating conditions, including different grid strengths, VSC power outputs and controller parameters, will be carried out to explore the potential unstable conditions, and examine the impact of these factors. Besides, a special example is also given to show the strictness of the proposed criterion over the classical one on stability judgment. Section 5 gives the simulation studies for the verification of the correctness of the net damping-based analysis. Lastly, conclusions are drawn in Section 6.

\section{Review of the Net Damping Criterion}

\subsection{The Classical Net Damping Criterion}

The net damping criterion is also called complex torque coefficients method [20]. This method is originally proposed to study the torsional interactions between generator shaft mode and the external grid electrical mode. As shown in Figure 1, the torque feedback loop is divided into two parts: the feedforward term $G_{\mathrm{m}}(\mathrm{s})$ representing the turbine's mechanical shaft dynamics, and the feedback term $G_{e}(s)$ denoting the dynamics of the rest power systems. It should be clarified that $G_{e}(s)$ includes the effect of the said generator's controls, e.g., voltage control and the power system stabilizer (PSS), and the impact of the power network and other generators. The frequency response functions for the feedforward and feedback terms are

$$
\begin{aligned}
\frac{1}{G_{m}(j \omega)} & =D_{m}(\omega)+j K_{m}(\omega) \\
G_{e}(j \omega) & =D_{e}(\omega)+j K_{e}(\omega)
\end{aligned}
$$


where $D$ and $K$ are denoted as the damping and spring coefficients; while the subscripts $m, e$ represent the mechanical and electrical subsystems. The net spring and damping coefficients are expressed as

$$
\begin{aligned}
& K(\omega)=K_{m}(\omega)+K_{e}(\omega) \\
& D(\omega)=D_{m}(\omega)+D_{e}(\omega)
\end{aligned}
$$

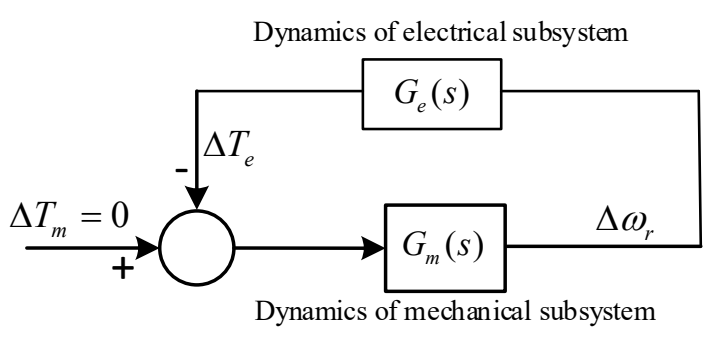

Figure 1. Representation of a power system by the linearized mechanical and electrical dynamic subsystems.

The criterion evaluates the net damping $D$ at the resonance frequency to judge stability. It claims that stability is guaranteed if $D>0$ holds at each resonance frequency $\omega$ satisfying $K(\omega)=0$. Plots of $K$ and $D$ versus frequency $\omega$ are generally utilized to capture a system's resonance conditions and damping characters visually.

\subsection{Limitations and the Complemental Criterion}

Literature [23] firstly proved that the criterion given by Canay primarily holds for weakly-damped oscillatory modes but cannot predict monotonic instability due to real positive poles and may falsely predict the stability of oscillatory modes with high damping ratios. Later, Harnefors [25] derived a connection between this criterion and the Nyquist criterion, and claims that an additional condition should be satisfied for stability, namely, damping of the low frequency range for loop gain exceeding unity should be positive. However, whether negative damping in this low frequency range means instability is not clarified. More recent works [26,27] proposed a similar net damping criterion, which is mapped from the Nyquist criterion in terms of the phase margin concept. The stability checking of this criterion is same as that of Bode criterion, which is known only feasible for minimum phase systems. However, it has been found that the dynamics of VSC interfaced type- 3 and -4 wind turbines can be non-minimum phase under weak-grid conditions [28,29].

Motivated by these works, recently, we proposed an alternative net damping criterion [24] dedicated for the interaction analysis of multi-VSC systems covering the frequency range of the outer power control dynamics. Different from the above criterion, the proposed criterion is strictly mapped from the Nyquist criterion in terms of the gain margin concept, so its judgment on stability is rigorous. And the framework for stability examination, by plotting spring/damping coefficients frequency response curves depicted in Section 2.1, can still be used, though a different way is utilized for stability determination. The theoretical derivation of this criterion is detailed in [24]. Hereby, we just put the procedures of the complemental criterion for stability checking:

Step (1) Applying the classical criterion, if the judgment result is instability then the system should be unstable; otherwise, if stability is predicted, go to Step (2);

Step (2) Checking the damping in the low frequencies close to zero, stability is guaranteed for positive damping, while negative damping indicates instability.

One point should be clarified that, checking the damping in the low frequencies in the vicinity of zero actually is equivalent to examining the clockwise encirclements of -1 at the frequency $\omega=0$ on the Nyquist contour of system open-loop transfer function [24]. Negative damping indicates 
one clockwise encirclement of -1 on the Nyquist contour at $\omega=0$, which corresponds to a real positive pole; so, instability is in the form of monotonic divergence. Therefore, compared to the results in [23], the complemental criterion can enlarge the applicability of the classical criterion for allowing the prediction of monotonic instability.

\section{System Modeling}

Figure 2a depicts the studied system comprised of two VSCs in parallel connection to a weak AC grid. The commonly used vector control strategy is adopted for the VSC's control. As shown in Figure $2 b$, it is constructed by two cascaded loops, i.e., the outer power controls and the inner current controls. The outer DC-link voltage control serves to maintain the DC voltage by regulating the active power flowing into the AC grid. The reactive power control channel is aimed to control the AC terminal bus voltage around the nominal. In addition, PLL synchronizes VSC's control system with the power grid by tracking the phase angle of the terminal bus voltage. Both the outputs of outer power controls and PLL provide references to the inner current controls; generally, the bandwidths of current controls are set ten times higher of the outer loops' [30]. Therefore, for the dynamic analysis of synchronization stability, the faster current control, circuit current dynamics and the switching process can be ignored. In other words, only the control dynamics of DC-link voltage control (DVC), AC voltage control (AVC) and PLL for each VSC are considered in the following modeling.

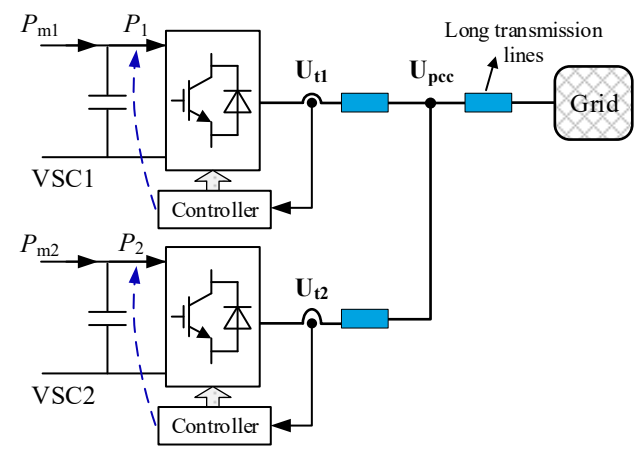

(a)

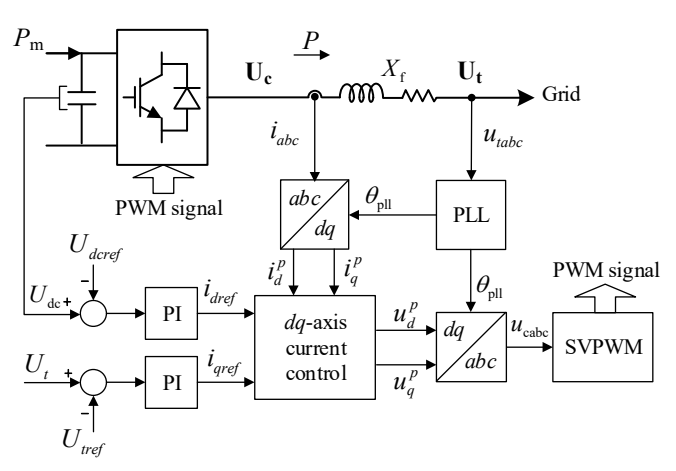

(b)

Figure 2. A system comprised of two VSCs in parallel connection to a weak grid: (a) circuit diagram; (b) VSC control system.

The equations for DVC and AVC of a VSC are

$$
\begin{gathered}
i_{\text {dref }}=\left(U_{d c}-U_{d c r e f}\right)\left(k_{p 1}+\frac{k_{i 1}}{s}\right) \\
i_{\text {qref }}=\left(U_{t}-U_{\text {tref }}\right)\left(k_{p 2}+\frac{k_{i 2}}{s}\right)
\end{gathered}
$$

The DC-link voltage is determined by its input and output powers as

$$
P_{m}-P=C U_{d c} \frac{d U_{d c}}{d t}
$$

The power flowed to the grid can be expressed as

$$
P=1.5\left(u_{t d} i_{d}+u_{t q} i_{q}\right)
$$

Figure 3a shows a typical second-order control scheme of PLL [1]. PLL functions to synchronize VSC's control system with the external power grid by tracking the phase angle of terminal voltage. The three-phase terminal voltage in Figure $3 a$ can be expressed by the polar form as $U_{\mathrm{t}} \angle \theta_{\mathrm{t}}$ 
in the synchronous frame. Referred to [31], the PLL model can be equivalently formulated in the synchronous frame as depicted in Figure 3b. The related equations are

$$
\begin{aligned}
& u_{\mathrm{tq}}^{\mathrm{p}}=u_{\mathrm{t}} \sin \left(\theta_{\mathrm{t}}-\theta_{\mathrm{pll}}\right) \\
& \theta_{\mathrm{pl}}=u_{\mathrm{tq}}^{\mathrm{p}}\left(k_{\mathrm{p} 3}+\frac{k_{\mathrm{i} 3}}{s}\right) \frac{1}{\mathrm{~s}}
\end{aligned}
$$

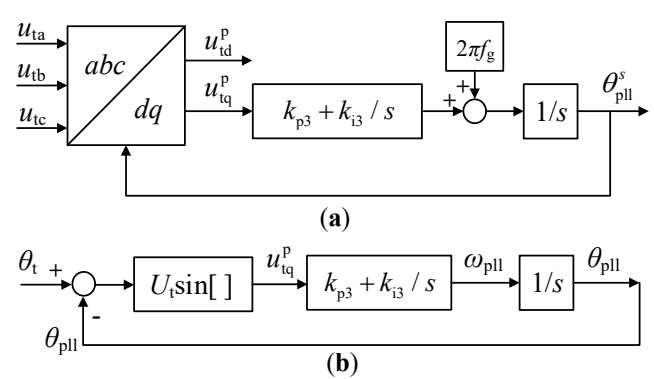

Figure 3. Diagram of PLL model [15]: (a) the actual structure of the PLL, (b) equivalent formulation in synchronous reference frame.

The corresponding linearized model for PLL is

$$
G_{p l l}(s)=\frac{\Delta \theta_{\mathrm{pll}}}{\Delta \theta_{\mathrm{t}}}=\frac{U_{\mathrm{t} 0} k_{\mathrm{p} 3} s+U_{\mathrm{t} 0} k_{\mathrm{i} 3}}{s^{2}+U_{\mathrm{t} 0} k_{\mathrm{p} 3} s+U_{\mathrm{t} 0} k_{\mathrm{i} 3}}
$$

The equations for the power network are

$$
\begin{gathered}
\mathbf{U}_{\mathbf{p c c}}-\mathbf{U}_{\mathbf{t} 1}=R_{1} \mathbf{I}_{1}+j X_{1} \mathbf{I}_{1} \\
\mathbf{U}_{\mathbf{p c c}}-\mathbf{U}_{\mathbf{t} 2}=R_{2} \mathbf{I}_{2}+j X_{2} \mathbf{I}_{2} \\
\mathbf{U}_{\mathbf{g}}-\mathbf{U}_{\mathbf{p c c}}=R_{g}\left(\mathbf{I}_{1}+\mathbf{I}_{2}\right)+j X_{g}\left(\mathbf{I}_{1}+\mathbf{I}_{2}\right)
\end{gathered}
$$

Generally, for the control of a VSC, the d-axis of the synchronous frame is chosen to coincide with its terminal bus voltage for its local control. However, for the network power flow calculations, the infinite bus voltage is usually selected as the reference of the global coordinate. So, the interface voltages and currents for the grid and the devices need to be transformed. Assuming the steady-state phase angle leading of a VSC's terminal voltage over the infinite bus voltage is $\theta_{0}$, then the relationships between the components (e.g., voltages and currents), as shown in Figure 4, in the global and local coordinate can be written as

$$
\left\{\begin{array}{l}
f_{x}=f_{d} \cos \theta_{0}-f_{q} \sin \theta_{0} \\
f_{y}=f_{d} \sin \theta_{0}+f_{q} \cos \theta_{0}
\end{array}\right.
$$



Figure 4. Transformation relations of a variable's dq-components between the global frame and the local frame [15]. 
Combining the control equations of each VSC and the grid circuit equations, the math model for the entire system can be built. Linearizing the math model around one equilibrium point, the small-signal model can be generated, which can be expressed as the diagram shown in Figure 5. It can be seen that each VSC can be regarded as a current source, while the grid side receives the current signals from the two VSCs and feeds back the power and voltage signals to VSC's control system.

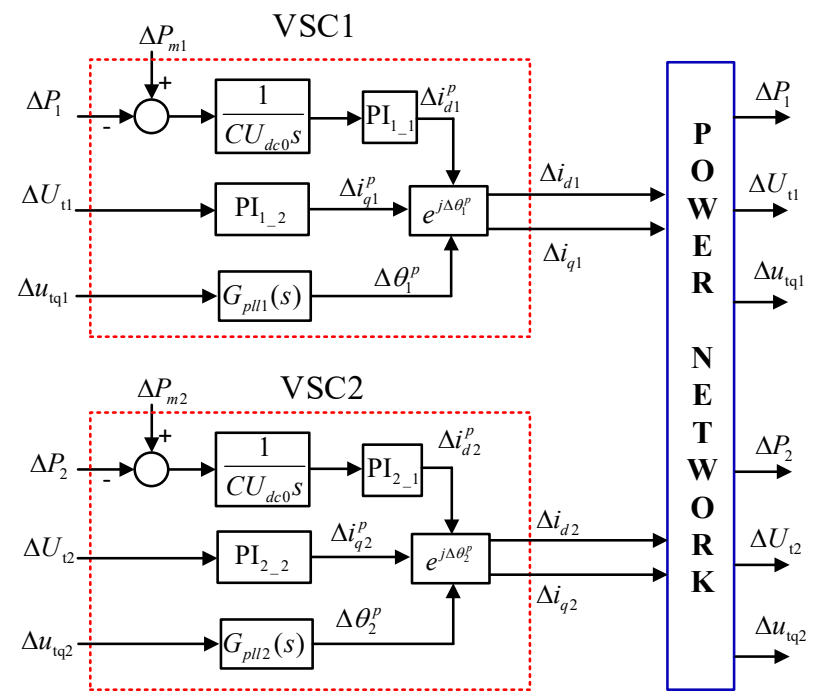

Figure 5. Block diagram of the small-signal model for a weak-grid-connected two-VSC system.

Due to the coupling of network power flows, the feedback active power of one VSC is affected not only by its own output current perturbations, but also influenced by the other VSC's current variations. Therefore, to study the impact of dynamic interactions, we can divide the feedback power to a VSC into two parts as shown in Figure 6: one caused by its own current changes through the self-effect path, the other part induced by the current variations of the other VSC via the interaction effect path. Besides, the transfer functions for $G_{1}(\mathrm{~s})$ and $G_{2}(\mathrm{~s})$ in Figure 6 can be derived from the model given in Figure 5, as the derivation process is tedious, and is accomplished by mathematical tools, the explicit expressions for the two complex transfer functions will not be listed herein.

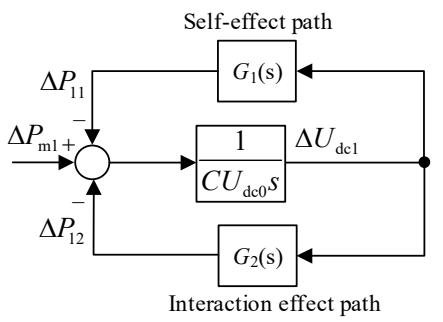

Figure 6. Diagram for illustration the feedback power components to VSC1 affected by itself and the interaction with VSC2.

In addition, as reviewed in Section 2, for the application of the net damping criterion in the electromechanical dynamics analysis of a multi-generator system, the closed-loop system is decomposed into the mechanical and electrical subsystems. Normally, the dynamics for the mechanical subsystem is described by the response of rotor speed to the torque imbalance between mechanical input and electrical output of said generator, while the rest of the system are all aggregated to the electrical subsystem. By contrast, multi-VSC systems involve the dynamic interactions between the DC and AC subsystems. Analogously, the DC subsystem dynamics can be represented by the DC-link voltage variations due to input-output power imbalance, namely, $G_{d c}(s)=1 /\left(C U_{d c 0} s\right)$, while 
the rest of the closed-loop system, including the effect of VSC controls and grid power flows, are all packaged into the $\mathrm{AC}$ subsystem $G_{\mathrm{ac}}(\mathrm{s})$. With $G_{d c}(s)$ and $G_{\mathrm{ac}}(\mathrm{s})$, net damping criterion can be utilized for stability analysis for the two-VSC system via the way described in Section 2. As shown in Figure 6, without considering the interactions of VSC2, $G_{\mathrm{ac}}(\mathrm{s})=G_{1}(\mathrm{~s})$; with the inclusion the interactions between the two VSCs, $G_{\mathrm{ac}}(\mathrm{s})=G_{1}(\mathrm{~s})+G_{2}(\mathrm{~s})$. In the following section, net damping characters for the two conditions will be comparably investigated to extract the impact the interactions of the two VSCs on closed-loop stability.

\section{Net Damping Based Stability Analysis}

Based on the proposed small-signal model, the net damping criterion will be utilized to examine the stability of the two-converter system at various conditions. In the following, the effects of grid strengths, VSC power outputs, and control parameters will be checked through sensitivity studies. The objective of this study is to identify the potential unstable conditions and locate the critical influence factors. For the case studies, the used parameters of the two VSCs are identical and listed in the Appendix A. The nominal power of the studied system is $2 \mathrm{MW}$, in which each VSC's base power is $1 \mathrm{MW}$.

\subsection{Effect of Grid Strength}

It is well known that weak-grid operation challenges the stability of VSC interfaced devices, such as the type- 3 and -4 wind turbines, and VSC-HVDC systems. Generally, grid strength is measured by the short-circuit ratio (SCR) [4]. In the case study, we assume both the two VSCs generate 1 pu active power. Figure 7 depicts the frequency response curves of spring coefficients and damping coefficients for SCR decreasing from 2.5 to 1.3. Figure 7a shows that, without considering the interaction with VSC2, the spring coefficients for VSC1 hardly change with grid strength variations, the frequency for $K_{1}(\omega)=0$ is around $24 \mathrm{rad} / \mathrm{s}$. Again, the damping changes for VSC1 for different grid stiffness is also quite small. In contrast, with the inclusion of the interaction with VSC2, as shown in Figure 7b, the spring coefficients curve $K(\omega)$ still changes little for grid strength variations, and the cross frequency for zero spring coefficients is about $26.5 \mathrm{rad} / \mathrm{s}$. However, significant reduction of damping is observed around this resonance frequency, this indicates that the interactions for the nearby two VSCs is detrimental for closed-loop stability. In particular, when SCR changes from 1.4 to 1.3, a negative damping region arises, and the damping at the resonance frequency ( $\omega=26.5 \mathrm{rad} / \mathrm{s})$ is negative, which means the loss of stability at this condition. As a conclusion, weaker grid connection will aggravate the interactions between the nearby VSCs, and such interactions are harmful for system stability.
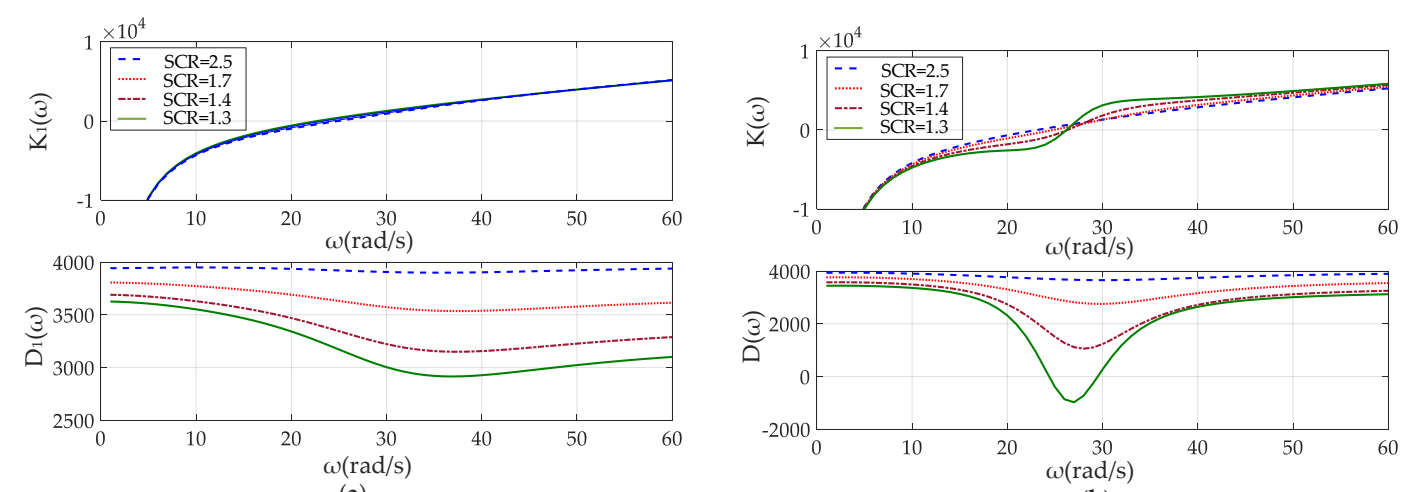

(a)

(b)

Figure 7. Frequency response curves of spring and damping coefficients under different grid strengths for (a) individual VSC1, (b) the entire system. 


\subsection{Effect of Loading Level}

This subsection investigates the impact of VSC output power $\mathrm{P}_{2}$ on the stability of the studied system. According to the aforementioned analysis, instability tends to happen at weak-grid conditions. So, we set SCR $=1.3$, and gradually increase the output powers of VSC2 to study the effect of loading level. Figure 8 gives the spring and damping coefficients analyses for VSC1 and the whole system, respectively. The results are close to those displayed in Figure 7. Variations of VSC2 output power changes system's operating points, but without considering the interaction within the two converters, the damping of VSC1 is sufficient and insensitive to this change, as depicted in Figure 8a. By contrast, the damping of the whole system gets degraded as the power of VSC2 increases. Additionally, it can be observed that loss of stability will occur at $\mathrm{P}_{2}=1 \mathrm{pu}$ for the negative damping at the resonance frequency. The analysis above shows that heavy loading conditions are also detrimental for system stability.
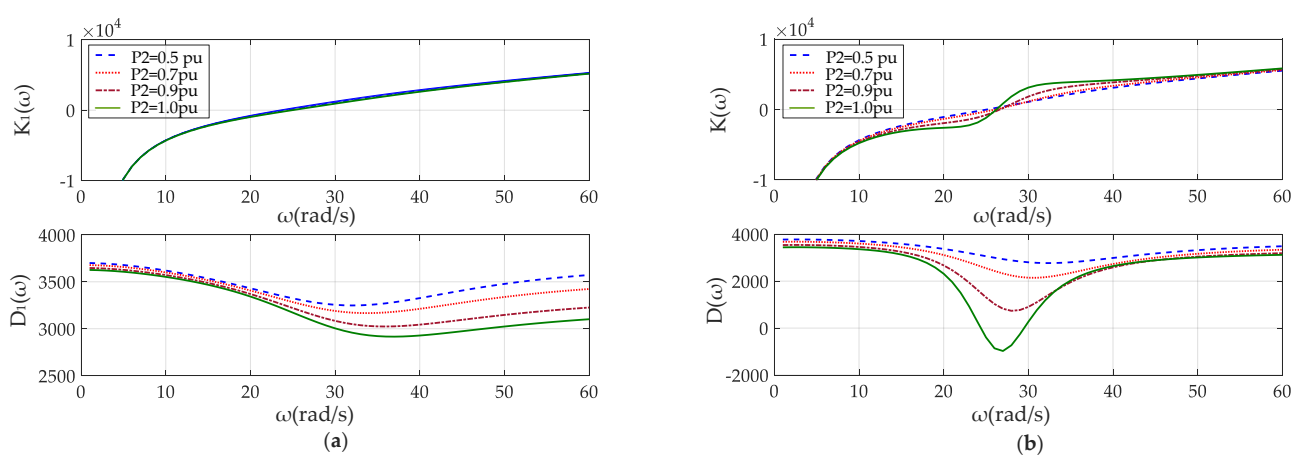

(b)

Figure 8. Frequency response curves of spring and damping coefficients under different VSC2 power outputs for (a) individual VSC1, (b) the entire system.

\subsection{Effect of AC Voltage Control Tuning}

This subsection checks the effect of AC voltage control. This case is configured as: $\mathrm{P}_{1}=\mathrm{P}_{2}=1 \mathrm{pu}$, $\mathrm{SCR}=1.3$. Figure 9 a gives the frequency response curves of the spring and damping coefficients for the bandwidths of AVC in VSC2 varying from $11.2 \mathrm{~Hz}$ to $1.4 \mathrm{~Hz}$. It can be found that with the reduction of AVC bandwidth, the resonance frequencies at $K(\omega)=0$ slightly decreases from around $30 \mathrm{rad} / \mathrm{s}$ to $24 \mathrm{rad} / \mathrm{s}$, while a negative damping region emerges and enlarges in this process, and loss of stability happens for $2.8 \mathrm{~Hz}$ and $1.4 \mathrm{~Hz}$ of the AVC bandwidth. The result of eigenvalue locus for the same condition is also given in Figure $9 \mathrm{~b}$ for comparison. System dominant mode moves to the right-half plane with the reduction of AVC bandwidth, and the modal frequency also decreases from $30 \mathrm{rad} / \mathrm{s}$ to around $24 \mathrm{rad} / \mathrm{s}$. This result is well accorded with that of the net damping analysis, which confirms that the analysis of net damping actually is focused on the behavior of system dominant mode. The case study hereby implies that faster AVC is preferable for the stability of this system under weak grids.
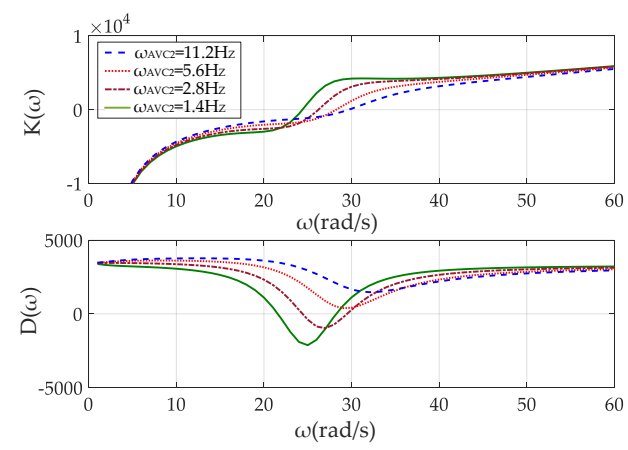

(a)



(b)

Figure 9. (a) Frequency response curves of spring and damping coefficients for the entire system under different bandwidths of AVC; (b) corresponding eigenvalue locus. 


\subsection{Effect of PLL Tuning}

This subsection examines the effect of PLL tuning. The same case configuration as Section 4.3 is adopted, and it should be said the bandwidth of PLL in VSC1 is $8.4 \mathrm{~Hz}$. Similarly, Figure 10a gives the result of net damping analysis for the bandwidth of PLL in VSC2 increasing from $4.2 \mathrm{~Hz}$ to $33.6 \mathrm{~Hz}$. It shows that the resonance frequency gradually increases along the process. An interesting finding is that a negative damping region first appears, then disappears in this duration. This means that system stability gets deteriorated, then restored. Additionally, the worst condition comes to that the PLL bandwidth in VSC2 is equal to that of in VSC1. Likewise, the corresponding eigenvalue locus is also depicted in Figure 10b for validation. It can be observed that the dominant mode first heads to the right-half plane, then goes back to the left side. The eigenvalue analysis results are also in good agreement with that of net damping analysis. This case study can conclude that the bandwidths of PLLs in the nearby VSCs are suggested to set separated for enhancing system stability.
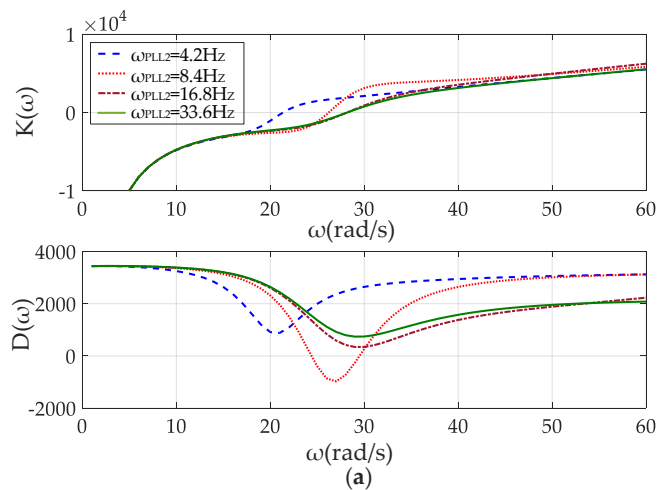

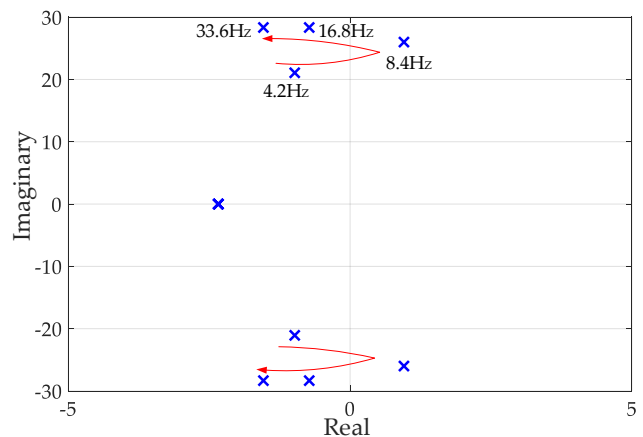

(b)

Figure 10. (a) Frequency response curves of spring and damping coefficients for the entire system under different bandwidths of PLL; (b) corresponding eigenvalue locus.

\subsection{An Unusual Case}

All the cases above show that the classical net damping criterion can well describe the impact of different parameters and subsystems on the system damping and give a correct prediction on stability. However, this criterion does not hold for all conditions, herein a counter example is offered. This case is set the same with that in Section 4.2 except that, the AC voltage controls of the two VSCs are disregarded, and instead, constant $q$-axis current controls, which can preserve the same operating point, are adopted.

Figure 11a shows that system total damping decreases with the increase of the output powers of VSC2, which implies a degradation of system stability. For the output power $\mathrm{P}_{2}$ varying from $0.5 \mathrm{pu}$ to $0.9 \mathrm{pu}$, the damping in the whole frequency range is positive, which means system is stable. The special condition comes to $P_{2}=0.95 \mathrm{pu}$. According to the classical criterion, there is no resonance frequency point corresponding to $K(\omega)=0$, so the system will be stable. However, this result is contradictory with the eigenvalue analysis given in Figure 11b, where a real positive pole occurs at $P_{2}=0.95 \mathrm{pu}$. So, the prediction of stability by the classical criterion is incorrect at this condition. By contrast, based on the complemental criterion, the damping at the low frequencies close to zero should also be checked. It can be observed that there exists a negative damping interval for the frequencies proximal to zero, so the system should be unstable. Besides, since there is only one real positive pole at this condition, the instability will be monotonic. This case study shows not only oscillations, monotonic instabilities can also happen for the two-VSC system under weak-grid conditions, in particular for the converters without grid voltage support functions. Again, the proposed complemental net damping criterion is more rigorous than the classical one, which is able to predict monotonic divergence. 

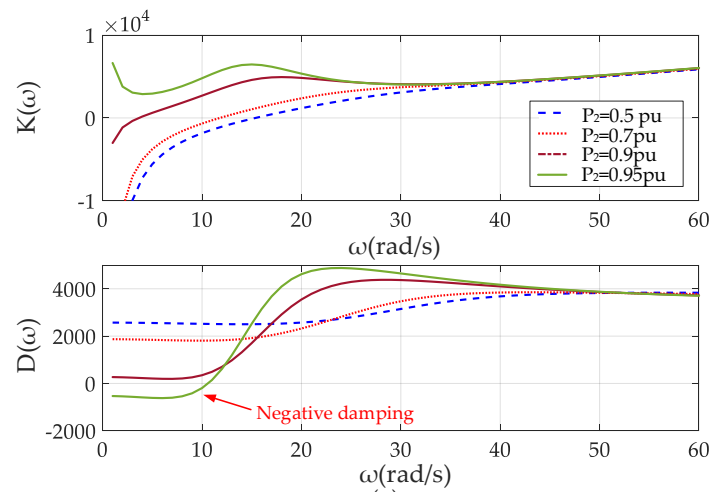

(a)

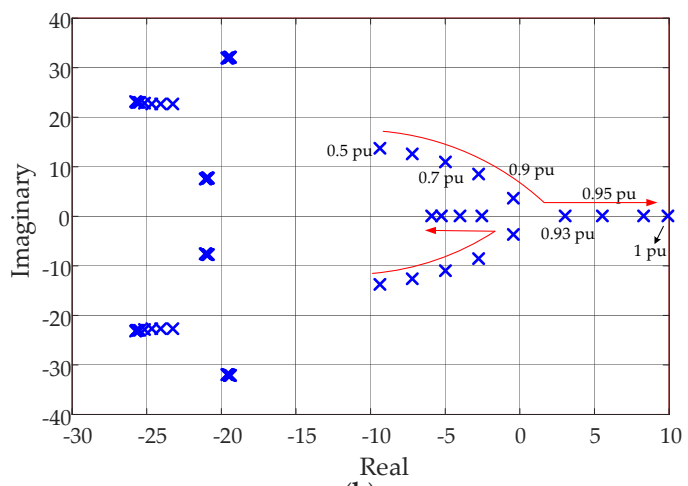

(b)

Figure 11. (a) Frequency response curves of spring and damping coefficients under different VSC2 power outputs when AVC of VSC1 and VSC2 is not included; (b) corresponding eigenvalue locus.

\section{Simulation Studies}

To verify the analysis above, a detailed model composed by two VSC interfaced type- 4 wind turbines parallel-connected to a weak grid is built in MATLAB/Simulink. The system configuration is the same as given in Figure 2, and the parameters used are identical with the case studies in Section 4. For all simulation set-ups, the pre-steady state is reached by proper tuning of control parameters, then at $\mathrm{t}=8 \mathrm{~s}$, a small disturbance, i.e., a $0.1 \%$ pu step-up of the active power reference on VSC1, is subjected, and the tuned control parameters are restored to the original settings. The responses of DC-link voltages in VSC1 and VSC2 are captured.

Figure 12 shows the responses of DC-link voltages in VSC1 and VSC2 for SCR varying from 2.5 to 1.3. It can be observed that system stability gets deteriorated with the reduction of grid strength, and instability occurs when SCR changes from 1.4 to 1.3. This result is well accorded with stability results by the net damping analysis in Section 4.1. Additionally, at SCR $=1.3$, the oscillation period of DC-link voltages observed is $0.232 \mathrm{~s}$, which corresponds to an angular frequency as $2 \pi / 0.232=27.08 \mathrm{rad} / \mathrm{s}$, which is quite close to the resonance frequency $26.5 \mathrm{rad} / \mathrm{s}$ analyzed by the net damping studies shown in Figure $7 \mathrm{~b}$. Therefore, this confirms that the net damping analysis can well capture the modal frequency and damping characters of system dominant oscillation mode. And it can be concluded that weak grid connection is harmful for the stability of a two-VSC system. Similarly, the effect of loading level is also checked as shown in Figure 13. With the increase of VSC2 output powers, the DC-link voltages in VSC1 and VSC2 gradually get oscillated and turn unstable at $\mathrm{P}_{2}=1.0 \mathrm{pu}$. Likewise, the simulation results also show good agreement with the net damping analysis in Section 4.2.

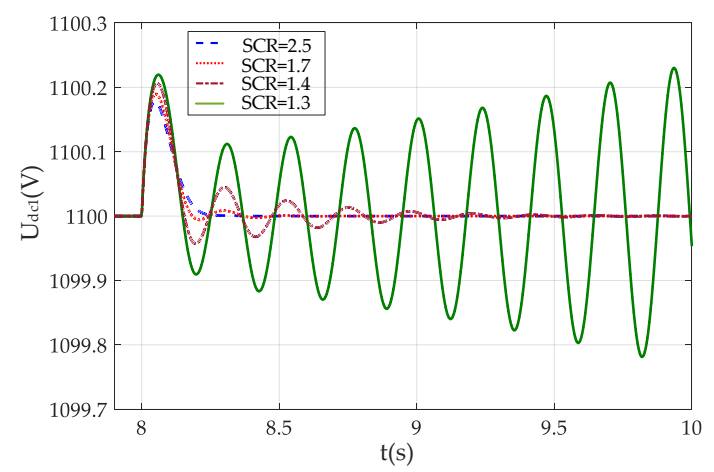

(a)

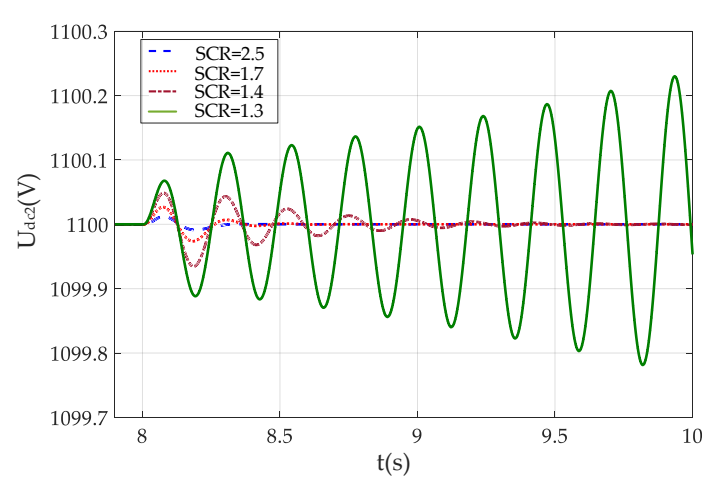

(b)

Figure 12. DC-link voltage responses under different grid strengths in (a) VSC1, (b) VSC2. 


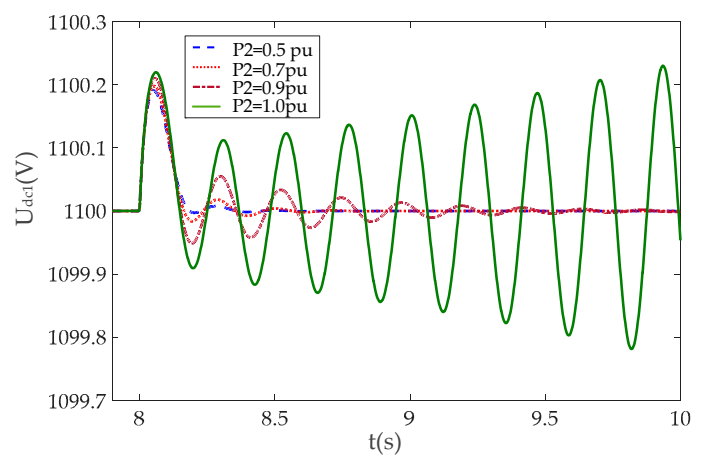

(a)

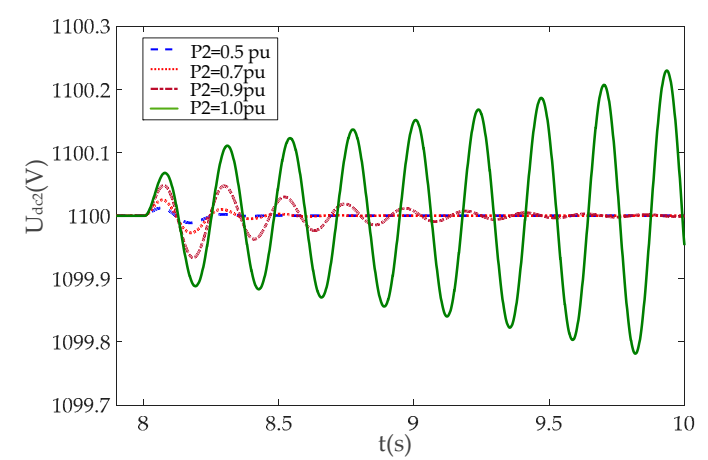

(b)

Figure 13. DC-link voltage responses under different VSC2' power outputs in (a) VSC1, (b) VSC2.

The effect of AVC is also simulated as depicted in Figure 14. The operating condition is set identical with that in Section 4.3 , in which $S C R=1.3, P_{1}=P_{2}=1 \mathrm{pu}$. The results indicate that with the decrease of AVC bandwidth in VSC2, the stability of system gets worse. When the bandwidth of AVC changes from $5.6 \mathrm{~Hz}$ to $2.8 \mathrm{~Hz}$, the DC-link voltage oscillations turn undamped. Further, with AVC bandwidth reduced to $1.4 \mathrm{~Hz}$, the oscillations get more severe. These simulation studies are also consistent with the results from net damping analysis and eigenvalue locus shown in Figure 9a,b. The results suggest that larger gain of AVC is beneficial for the stability of the studied system.

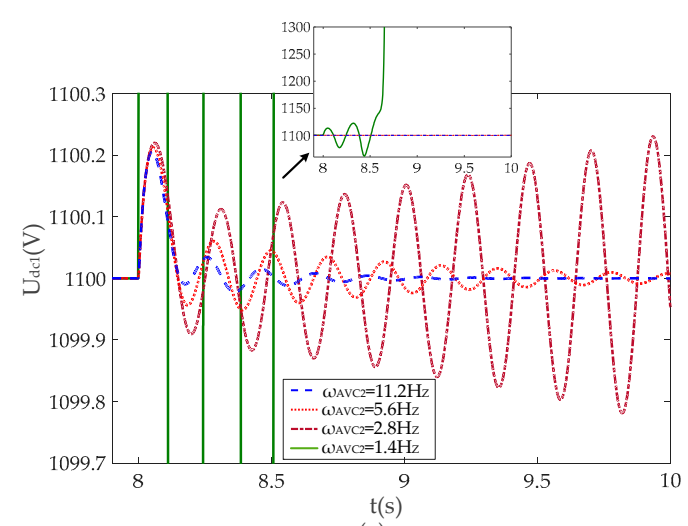

(a)

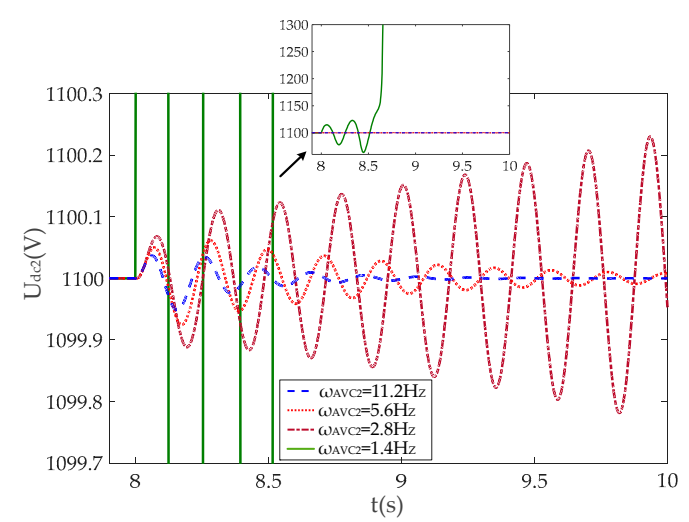

(b)

Figure 14. DC-link voltage responses under different VSC2's AVC bandwidths in (a) VSC1, (b) VSC2.

The impact of PLL tuning is checked, and the simulation results are shown in Figure 15. The set-up conditions are the same with that in Section 4.4, where the PLL bandwidth in VSC1 is fixed to $8.4 \mathrm{~Hz}$, and varied PLL bandwidth in VSC2 is examined. The results show that when PLL bandwidth in VSC2 increases from $4.2 \mathrm{~Hz}$ to $8.4 \mathrm{~Hz}$, approaching the PLL bandwidth in VSC1, the DC-link voltage oscillations turn from stable to unstable. However, as the PLL bandwidth in VSC2 further increases, system stability gets restored. The simulation results show that when the PLL bandwidths in the two VSCs are close, system stability gets deteriorated. These results are also accorded with the net damping analysis and eigenvalue analysis given in Figure 10a,b. This study recommends that separating the bandwidths of the PLL of the two VSCs is useful to enhance system stability.

Also, a simulation study was done to validate the analysis given in Section 4.5. For the setup of this simulation case, the AVC is included for the sake of reaching a steady state before $t=8 \mathrm{~s}$; then at $\mathrm{t}=8 \mathrm{~s}$, the AVC is excluded, and replaced by a constant $q$-axis current control, whose reference is assigned by keeping the same power flow conditions. The results in Figure 16 show that system stability is deteriorated with the increase of output powers of VSC2. When $\mathrm{P}_{2}$ increases from $0.9 \mathrm{pu}$ to $0.95 \mathrm{pu}$, system turns from stable to unstable, this is well accorded with the net damping and eigenvalue analysis shown in Figure 11. In addition, at $P_{2}=0.95 \mathrm{pu}$, monotonic divergence is observed, which 
implies the instability is induced by a real positive pole, rather than a pair of complex poles. This is also consistent with the analysis given in Section 4.5. This simulation study indicates that monotonic instabilities can also occur at weak-grid conditions, in particular for the converters not equipped with grid voltage control functions.

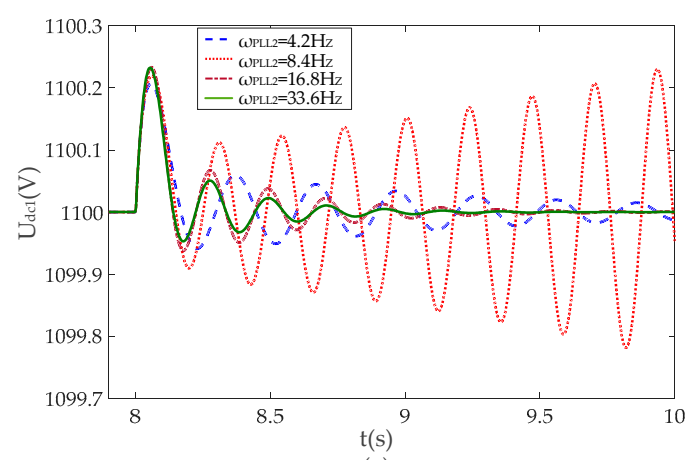

(a)

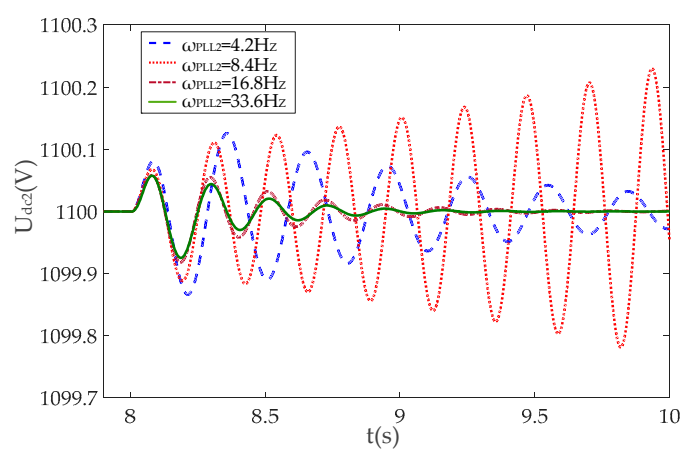

(b)

Figure 15. DC-link voltage responses under different VSC2's PLL bandwidths in (a) VSC1, (b) VSC2.

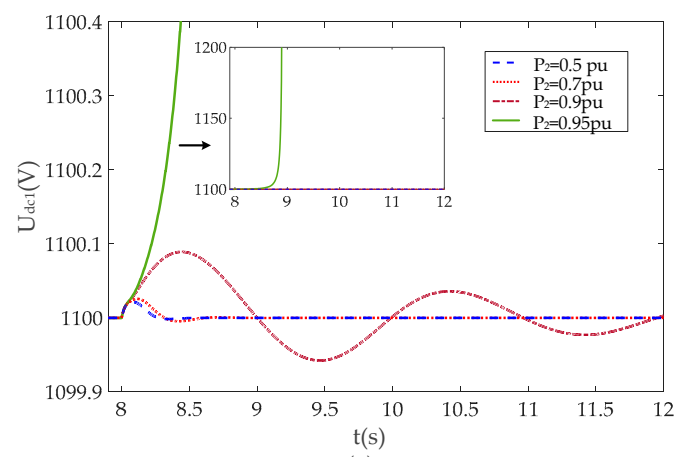

(a)

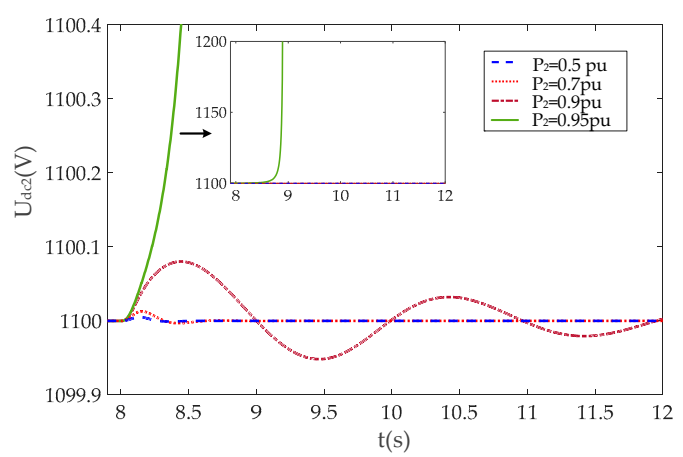

(b)

Figure 16. DC-link voltage responses under different VSC2' power outputs for open-loop AVC conditions in (a) VSC1, (b) VSC2.

\section{Conclusions}

In this paper, the synchronization stability of a system comprised by two VSCs in parallel connected to a weak AC grid is evaluated. A reduced-order small-signal model dedicated for the analysis of synchronization control time scale dynamics is proposed. The model neglects the fast dynamics of inner current controls for simplicity of analysis but can allow for the possible dynamic interactions between PLL and the outer power controls. An improved net damping criterion is used as the analysis tool to explore the potential instability conditions and make out the impact of interactions between the nearby converters. Compared to the conventional net damping criterion, the used criterion is more rigorous, which is able to predict monotonic instability. Various case studies show that the stability of the two-VSC system get worse with the decrease of grid strength, as well as with the increase of loading level. Additionally, the identified instabilities can be both in the form of oscillations or monotonic divergence. In particular, the latter is more likely to happen for the converters without grid voltage regulation functions. Besides, the net damping-based sensitivity studies indicate that higher bandwidths of AC voltage control and separation of PLL bandwidths in different VSCs are both beneficial to improve the stability of parallel converters in close proximity. Also, this frequency domain net damping analysis can offer some insight on designing reshaping damping techniques for stability enhancement, which will be studied in the near future. Again, the proposed net damping criterion is also promising for investigating the dynamic interactions of other AC-DC networks, e.g., the MTDC systems. 
Author Contributions: Conceptualization, D.W.; methodology, D.W., X.Z. and Y.H.; software, D.W.; validation, D.W. and X.Z.; investigation, Y.H. and X.Z.; writing—original draft preparation, D.W.; writing—review and editing, D.W., L.Y., S.L. and W.H.; supervision, Y.H. and C.W.; project administration, X.Z. and D.W.; funding acquisition, L.Y. and D.W. All authors have read and agreed to the published version of the manuscript.

Funding: This project is supported by Science and Technology Project of China Southern Power Grid Company Limited (056200KK52190009) and Research Start-Up Fund of Wuhan University of Technology under Grant 40120531.

Conflicts of Interest: The authors declare no conflict of interest.

\section{Nomenclature}

$\begin{array}{ll}U_{\mathrm{t}}, \theta_{\mathrm{t}} & \text { Terminal voltage magnitude and phase } \\ \theta_{\mathrm{pll}}, \omega_{\mathrm{pll}} & \text { PLL output angle and frequency } \\ U_{\mathrm{dc}}, C & \text { DC-link voltage and capacitance } \\ \text { PCC } & \text { Point of common connection } \\ X_{1}, X_{2} & \text { Reactance between terminal bus of VSC1 and VSC2 to the PCC } \\ X_{g} & \text { Reactance between PCC and the infinite bus } \\ k_{p}, k_{i} & \text { Proportion and integral control gain } \\ L_{g} & \text { Transmission line inductance between the PCC and the infinite bus } \\ L_{i} & \text { Equivalent line inductance between VSC terminal bus and the PCC } \\ \text { Subscripts: } & \\ 0 & \text { Steady-state value } \\ d, q & \text { Synchronous rotating reference frame signal d-axis and q-axis } \\ x, y & \text { components } \\ r e f & \text { Global reference frame signal } x \text {-axis and } y \text {-axis components } \\ \text { Superscript: } & \text { Reference signal } \\ p & \text { Components in PLL rotating frame }\end{array}$

\section{Appendix A}

System circuit parameters:

\begin{tabular}{cccc}
\hline$S_{\text {base }}=1 \mathrm{MW}$ & \multicolumn{2}{c}{$U_{\text {base }}=690 \mathrm{~V}($ phase to phase RMS value $)$} \\
$\omega_{\text {base }}=2 \pi f_{\text {base }}$ & $f_{\text {base }}=50 \mathrm{~Hz}$ & $U_{\text {dcref }}=1100 \mathrm{~V}$ & $\mathrm{C}=0.09 \mathrm{~F}$ \\
$L_{f}=0.1$ p.u. & $L_{1}=0.1$ p.u. & $L_{2}=0.1$ p.u. & \\
\hline
\end{tabular}

Controller parameters values:

\begin{tabular}{ccc}
\hline DC-link voltage control & $k_{\mathrm{p} 1}=5$ & $k_{\mathrm{i} 3}=60$ \\
AC voltage control & $k_{\mathrm{p} 2}=1$ & $k_{\mathrm{i} 2}=100$ \\
Phase-locked loop & $k_{\mathrm{p} 3}=40$ & $k_{\mathrm{i} 3}=1400$ \\
Current control & $k_{\mathrm{p} 4}=1$ & $k_{\mathrm{i} 4}=50$ \\
\hline
\end{tabular}

\section{References}

1. Teodorescu, R.; Liserre, M.; Rodriguez, P. Grid Converters for Photovoltaic and Wind Power Systems; Wiley IEEE Press: Piscataway, NJ, USA, 2011.

2. Thacker, T.; Boroyevich, D.; Burgos, R.; Wang, F. Phase-locked loop noise reduction via phase detector implementation for single phase systems. IEEE Trans. Ind. Electron. 2011, 58, 2482-2490. [CrossRef]

3. Zhang, H.; Harnefors, L.; Wang, X.; Gong, H.; Hasler, J.P. Stability Analysis of Grid-Connected Voltage-Source Converters Using SISO Modeling. IEEE Trans. Power Electron. 2019, 34, 8104-8117. [CrossRef]

4. Li, S.; Yan, Y.; Yuan, X. SISO Equivalent of MIMO VSC-Dominated Power Systems for Voltage Amplitude and Phase Dynamic Analyses in Current Control Timescale. IEEE Trans. Energy Convers. 2019, 34, 1454-1465. [CrossRef] 
5. Yuan, H.; Yuan, X.; Hu, J. Modeling of grid-connected VSCs for power system small-signal stability analysis in DC-link voltage control timescale. IEEE Trans. Power Syst. 2017, 32, 3981-3991. [CrossRef]

6. Huang, Y.; Zhai, X.; Hu, J.; Liu, D.; Lin, C. Modeling and Stability Analysis of VSC Internal Voltage in DC-Link Voltage Control Time Scale. IEEE J. Emerg. Sel. Top. Power Electron. 2018, 6, 16-28. [CrossRef]

7. Zhou, J.; Ding, H.; Fan, S. Impact of short-circuit ratio and phase-locked-loop parameters on the small-signal behavior of a VSC-HVDC converter. IEEE Trans. Power Deliv. 2014, 29, 2287-2296. [CrossRef]

8. Alawasa, K.M.; Mohamed, Y.A.; Xu, W. Active Mitigation of Subsynchronous Interactions Between PWM Voltage-Source Converters and Power Networks. IEEE Trans. Power Electron. 2014, 29, 121-134. [CrossRef]

9. Wen, B.; Dong, D.; Boroyevich, D.; Burgos, R.; Mattavelli, P.; Shen, Z. Impedance-Based Analysis of Grid-Synchronization Stability for Three-Phase Paralleled Converters. IEEE Trans. Power Electron. 2016, 31, 26-38. [CrossRef]

10. Li, C.; Burgos, R.; Wen, B.; Tang, Y.; Boroyevich, D. Stability Analysis of Power Systems with Multiple STATCOMs in Close Proximity. IEEE Trans. Power Electron. 2020, 35, 2268-2283. [CrossRef]

11. Yuan, Z.; Du, Z.; Li, G. Analysis on the dynamic behaviours and interactions of VSC-MTDC grid. IET Gener. Transm. Distrib. 2018, 12, 1756-1764. [CrossRef]

12. Zheng, W.; Hu, J.; Yuan, X. Modeling of VSCs Considering Input and Output Active Power Dynamics for Multi-Terminal HVDC Interaction Analysis in DC Voltage Control Timescale. IEEE Trans. Energy Convers. 2019, 34, 2008-2018. [CrossRef]

13. Huang, Y.; Wang, D.; Shang, L.; Zhu, G.; Tang, H.; Li, Y. Modeling and stability analysis of DC-link voltage control in multi VSCs with integrated to weak grid. IEEE Trans. Energy Convers. 2017, 32, 1127-1138. [CrossRef]

14. Rosso, R.; Andresen, M.; Engelken, S.; Liserre, M. Analysis of the interaction among power converters through their synchronization mechanism. IEEE Trans. Power Electron. 2019, 34, 12321-12332. [CrossRef]

15. Wang, D.; Huang, Y.; Liao, M.; Zhu, G.; Deng, X. Grid-synchronization stability analysis for multi DFIGs connected in parallel to weak AC grids. Energies 2019, 12, 4361. [CrossRef]

16. Turner, R.; Walton, S.; Duke, R. A case study on the application of the Nyquist stability criterion as applied to interconnected loads ans sources on grids. IEEE Trans. Ind. Electron. 2013, 60, 2740-2749. [CrossRef]

17. Wang, X.; Blaabjerg, F.; Loh, P.C. Passivity-based stability analysis and damping injection for multi paralleled VSCs with LCL filters. IEEE Trans. Power Electron. 2017, 32, 8922-8935. [CrossRef]

18. Harnefors, L.; Wang, X.; Yepes, A.G.; Blaabjerg, F. Passivity-based stability assessment of grid-connected VSCs-An overview. IEEE J. Emerg. Sel. Top. Power Electron. 2016, 4, 116-125. [CrossRef]

19. Harnefors, L. Modeling of three-phase dynamic systems using complex transfer functions and transfer matrices. IEEE Trans. Ind. Electron. 2007, 54, 2239-2248. [CrossRef]

20. Canay, I.M. A novel approach to the torsional interaction and electrical damping of synchronous machine, part I: Theory. IEEE Trans. Power App. Syst. 1982, PAS-101, 3630-3638. [CrossRef]

21. Canay, I.M. A novel approach to the torsional interaction and electrical damping of synchronous machine, part II: Application to an arbitrary network. IEEE Trans. Power App. Syst. 1982, PAS-101, 3639-3647. [CrossRef]

22. Stamatiou, G.; Bongiorno, M. Stability analysis of two-terminal VSC-HVDC systems using the net-damping criterion. IEEE Trans. Power Deliv. 2016, 31, 1748-1756. [CrossRef]

23. Tabesh, A.; Iravani, R. On the application of the complex torque coefficients method to the analysis of torsional dynamics. IEEE Trans. Energy Convers. 2005, 20, 268-275. [CrossRef]

24. Wang, D.; Hou, Y.; Hu, J. Net Damping Criterion for Stability Analysis of Grid-Tied VSCs in DC Voltage Control Timescale. CSEE J. Power Energy Syst. 2020. In early access. [CrossRef]

25. Harnefors, L. Proof and application of the positive-net-damping stability criterion. IEEE Trans. Power Syst. 2011, 26, 481-482. [CrossRef]

26. Sainz, L.; Cheah-Mane, M.; Monjo, L.; Liang, J.; Gomis-Bellmunt, O. Positive-net-damping stability criterion in grid-connected VSC systems. IEEE J. Emerg. Sel. Top. Power Electron. 2017, 5, 1499-1512. [CrossRef]

27. Cheah-Mane, M.; Sainz, L.; Liang, J.; Jenkins, N.; Ugalde-Loo, C.E. Criterion for electrical resonance stability of offshore wind power plants connected through HVDC links. IEEE Trans. Power Syst. 2017, 32, 4579-4589. [CrossRef]

28. Fan, L.; Miao, Z. An explanation of oscillations due to wind power plants weak grid interconnection. IEEE Trans. Sustain. Energy. 2018, 9, 488-490. [CrossRef] 
29. Wang, D.; Liang, L.; Hu, J.; Chang, N.; Hou, Y. Analysis of low-frequency stability in grid-tied DFIGs by nonminimum phase zero identification. IEEE Trans. Energy Convers. 2018, 33, 716-729. [CrossRef]

30. Harnefors, L.; Bongiorno, M.; Lundberg, S. Input-admittance calculation and shaping for controlled voltage-source converters. IEEE Trans. Ind. Electron. 2007, 54, 3323-3334. [CrossRef]

31. He, W.; Yuan, X.; Hu, J. Inertia provision and estimation of PLL-based DFIG wind turbines. IEEE Trans. Power Syst. 2017, 32, 510-521. [CrossRef]

C 2020 by the authors. Licensee MDPI, Basel, Switzerland. This article is an open access article distributed under the terms and conditions of the Creative Commons Attribution (CC BY) license (http://creativecommons.org/licenses/by/4.0/). 